\title{
Fabrication of Binary Diffractive Lenses and the Application to LED Lighting for Controlling Luminosity Distribution
}

\author{
Atsushi Motogaito, Kazumasa Hiramatsu \\ Division of Electrical and Electronic Engineering, Graduate School of Engineering, Mie University, Tsu, Japan \\ Email: motogaito@elec.mie-u.ac.jp
}

Received November 29, 2012; revised December 30, 2012; accepted January 6, 2013

\begin{abstract}
We designed and fabricated two types of binary diffractive lenses using Electron beam lithography (EBL) on optical films film for controlling LED light. In the case of the binary diffractive convex lens with 2-mm focal length, it is possible to control the luminous intensity distribution. To improve the diffraction efficiency and realize a thin LED light source, the binary diffractive lenses with $100-\mu \mathrm{m}$-order focal length are effective. Furthermore we fabricated and characterized the binary diffractive concave lenses for application in LED lighting. It is found that white-light LEDs are strongly diffused by using the binary diffractive concave lenses.
\end{abstract}

Keywords: Light Emitting Diode (LED); Luminosity Distribution; Binary Diffractive Lens; Electron Beam Lithography (EBL); Optical Film

\section{Introduction}

Light-emitting diodes (LEDs) are used increasingly as lighting devices because their efficiency has recently been improved. For such application, it is desirable to miniaturize LEDs for smaller lighting devices and control their luminosity. Conventional oval-lamp type LEDs cannot meet these requirements because their lens is approximately $5 \mathrm{~mm}$ in size and their light distribution is determined by the lens shape. To use LEDs as lighting devices, their luminosity distribution must be controlled because the LED itself is a point source. By using shortfocal-length $(\sim 100 \mu \mathrm{m})$ lenses, the spatial distribution of LED light can be controlled and a thin LED light source can be produced.

Two types of lenses can focus light: an optical lens that uses refraction and a diffractive lens that uses diffraction. The focal length of the diffractive lens is controlled by the structure of the lens, which suggests that the focal length may be independent of the curvature and index of refraction. Thus, diffractive lenses can be used as UV optical elements or thin optical elements [1].

In 1875 , Solet invented the zone plate, which was the first diffractive lens [2]. To improve light efficiency, Lesem et al. invented the kinoform $[3,4]$. Then, diffractive optical elements with multilevel gratings and step-like cross sections were developed, driven by the development of binary-optics technology based on computer- aided design and very-large-scale integrated technology [5]. By controlling the structure of multilevel gratings, an optical effect that is almost identical to that exploited by the kinoform can be obtained [6,7]. Subwavelength structures (SWSs), which are equivalent to blazed structures, were proposed by Lalanne et al., [8,9] and are essentially binary SWSs fabricated by converting from Fresnel lenses. By using these structures, achromatic diffractive lenses were obtained [10].

Diffractive lenses are typically used with monochromatic light sources such as lasers because the focal length of the lens depends on wavelength. However, white-light LEDs emit over a wide spectral range that contains the spectrum of blue LEDs and that of yellow phosphor. Therefore, it is difficult to use diffractive lenses to control the diffusion of white-light LEDs. However, despite these difficulties, Okada et al. used a diffractive lens in a white-light LED imaging device [11]. Their device is composed of two materials. The first has a low index of refraction and low Abbe number, and the second is a nanocomposite material which has a high index of refraction and high Abbe number.

In this paper, the control of luminosity distribution of LED by using the binary diffractive lenses is described. Two types of the binary diffractive lenses are demonstrated. The one is the binary diffractive convex lens for focusing LED light $[1,12-14]$ and the other is the binary diffractive concave lens for diffusing LED light. These 
lensesare are fabricated by electron beam lithography (EBL) because the binary diffractive lens contains many submicron-sized structures. By using nanoimprint lithography (NIL) or photolithography, these structures can be easily fabricated at low cost and over large areas (for example more than $10 \mathrm{~mm} \times 10 \mathrm{~mm}$ ). However, EBL does not use any molds or masks, and therefore, it is an attractive alternative that merits examination in detail as a way to optimize structures.

\section{The Procedure of Design and Experiments}

\subsection{Design of the Binary Diffractive Lenses}

The design of fabricated binary diffractive lenses was based on the design of micro-Fresnel lenses. In this study, a part of the two-level zone plates with a pattern of lines and spaces was fabricated. The radius of the $m$ th zone, $r_{m}$, is given as

$$
r_{m}=2 m f \lambda_{0}+\left(m \lambda_{0}\right)^{2}
$$

where $f$ is the focal length of the designed lens and $l$ is the dominant wavelength. Equation (1) is based on the imaging theory of the diffractive lens [15].

The $m$ th period of this lens, $d_{m}$, is determined by $r_{m}-$ $r_{m-1}$. In the period $\mathrm{dm}$, the blazed structure is approximated by a step-like structure with $n$ steps and then the step-like structure is converted to a relief structure by a duty ratio of height $t_{i}=1-h\left(x_{\mathrm{i}}\right) / h_{\max }$ in each interval $g$ (Figure 1(a)). In the interval, the width of the air is given by $g * t_{i}$. In the binary diffractive lens, $N$ is the number of relief structures in a period. Examples of the structures are shown in Figure 1(b) and the complete structure of this lens is shown in Figure 1(c). To achieve divergence in a binary diffractive concave lens, the step-like structures are inverted (i.e., "off" and "on" are inverted), as shown in Figure 1(d). To confirm the characteristics of this lens, the finite domain time difference (FDTD) simulation is carried out.

\subsection{Fabrication and Characterization of the Binary Diffractive Lenses}

The substratesare made up of 125- $\mu$ m-thick poly (ethylene terephthalate) (PET) films or $188-\mu \mathrm{m}$-thick cyclic olefin polymer (COP) films. Before spin coating the EB resist, hexamethyldisilazane (HDMS) was spin coated on the surface of the PET film to improve the adherence between the PET film and the EB resist. The surface was spin coated with an EB positive resist followed by prebaking. Then, the charge-up prevention treatment was spin coated on the EB resist.

The EBL system (Crestec CABL-8000) was equipped with a $\mathrm{ZrO} / \mathrm{W}$ thermal field emission cathode. The acceleration voltage was $30 \mathrm{kV}$; electrons accelerated by this (a)

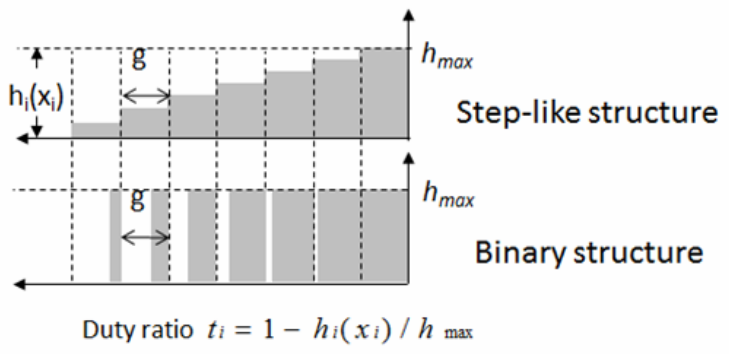

(b)
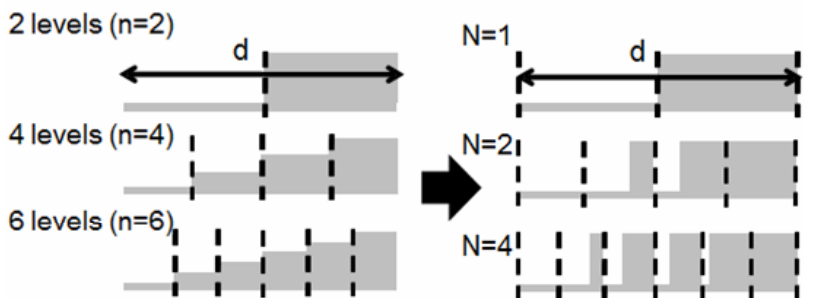

(c)

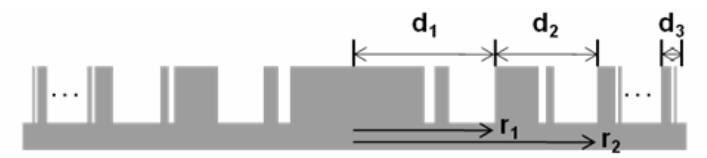

(d)

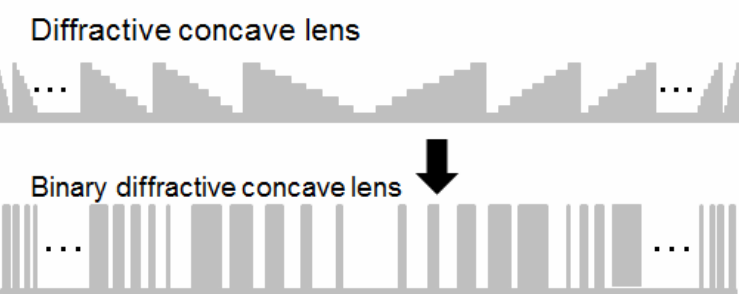

Figures 1. Structure of binary diffractive lens: (a) Conversion of step-like structure to binary structure; (b) Examples of structures; (c) Complete structure. In Figure 4(a), the height of an intermediate step (e.g., the third or fourth step) as " $h\left(x_{i}\right)$ " for the same step as step $I$; (d) Conversion method of the binary diffractive concave lens.

voltage were able to penetrate the resist. After exposure, the resist was developed, resulting in the binary diffractive lens. The size of the pattern for the binary diffractive lens was between $0.1 \times 0.1$ and $2.0 \times 2.0 \mathrm{~mm}^{2}$. The optimum characteristics of the diffractive lenses fabricated by EBL, such as period, width and height, are useful for fabricating the moulds for a thermal-type NIL. Optical characterizations, such as far-field transmitted intensity distribution, luminosity distribution of LED and so on, are done with a red laser $(\lambda=635 \mathrm{~nm})$, a green laser $(\lambda=$ $532 \mathrm{~nm})$, a green $\operatorname{LED}(\lambda=566 \mathrm{~nm})$ and a white-light LED.

\section{Results and Discussion}

\subsection{Binary Diffractive Convex Lenses}

The binary diffractive convex lens with 2-mm focal 
length was fabricated on the PET film. Figure 2 shows the scanning electron microscopy (SEM) image of the fabricated binary diffractive lens on the PET film. The diffractive lens having width almost same as that of the designed lens was obtained.

Optical characterization of the fabricated binary diffractive lens was carried out. The luminous intensity distribution of the LED $(\lambda=566 \mathrm{~nm})$ for the binary diffractive lens was characterized using a luminous intensity distribution system (Asahi Spectra IMS5000-LED). The fabricated lens was then mounted on the LED chip and spectral irradiance in the vertical direction was measured; Figure 3 shows the distribution of the irradiance. Most of the LED light was focused, as shown in Figure 3(a); the light distribution angle became narrow $\left(30^{\circ}\right)$ using the binary diffractive lens. As shown in Figure 3(b), spectral irradiance around $0^{\circ}$ with this lens was 1.5 times higher than that without the lens. On the other hand, two side peaks in these data were observed and believed to be due to light escaping from the fabricated binary diffractive lens. From these results, it is clear that the luminous intensity distribution can be controlled using this type of lens.

Although the binary diffractive lens was effective in controlling the luminous intensity, diffraction efficiency was reduced when the diffraction angle was decreased $[8$, 10]. Furthermore, the focal length of the fabricated binary diffractive lens is $2 \mathrm{~mm}$. In order to realize a thin LED light source, the focal length has to be shorter. In this section, to improve the diffraction efficiency and shorten the focal length, we designed the binary diffractive convex lens with $150-\mu \mathrm{m}$ focal length. A binary diffractive lens with a focal length of approximately $150 \mu \mathrm{m}$ was designed and light propagation of the plane wave

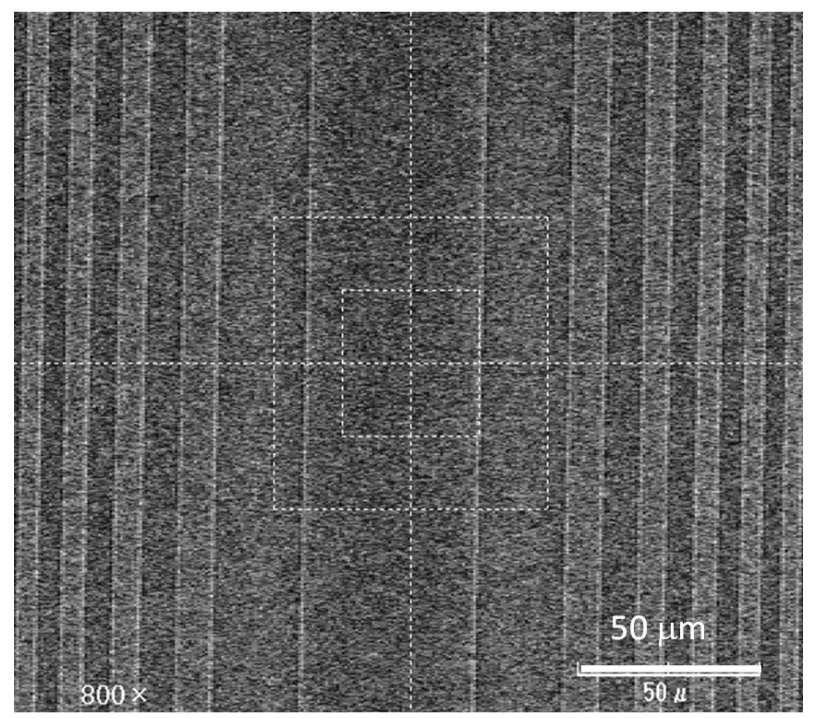

Figure 2. SEM image of fabricated binary diffractive convex lens on a PET film.
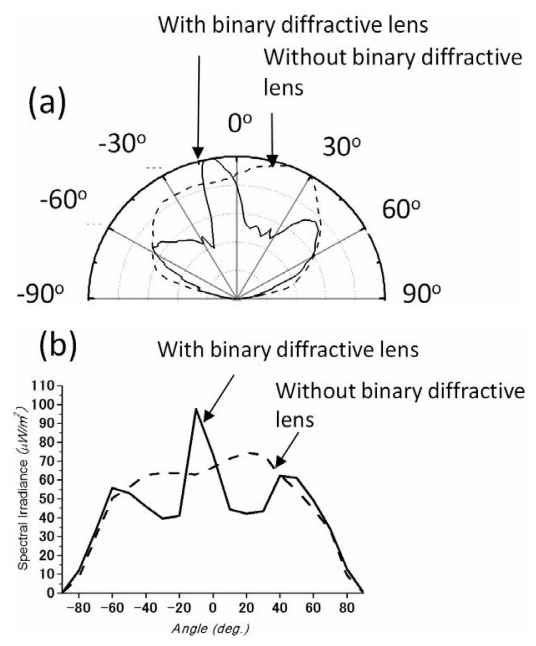

Figures 3. Distribution of spectral irradiance. (a) Angle dependence of normalized spectral irradiance; (b) Angle dependence of the absolute value of spectral irradiance.

was simulated by the finite domain time difference (FDTD) method. Figure 4 shows the field intensity distributions for TE polarization of the binary diffractive lens. The simulation parameters were $\lambda=632 \mathrm{~nm}, n=$ 1.575 (refractive index of the PET film), and $n_{0}=1.0$ (refractive index of air). The value of the period in part of the fringe was smaller than that in the center. The designed lens was placed along the $x$-axis $(z=0)$. The light was incident from $z=0$ to the $+z$ direction, resulting in the light being focused at $x=0 \mu \mathrm{m}$ and $z=140 \mu \mathrm{m}$. After focusing, the light was spread with time because of diffraction. Therefore, a binary diffractive lens with a micrometer-order focal wavelength is expected to provide a small and thin light source for controlling the luminous intensity distribution. On the basis of the results of section 3.1, we speculated that the LED light can be focused at $140 \mu \mathrm{m}$.

The binary diffractive lens with a $150-\mu \mathrm{m}$ focal length was fabricated; its size was $100 \times 100 \mu \mathrm{m}^{2}$ and thickness was $570 \mathrm{~nm}$, as measured by ellipsometry. Figure 5 shows the SEM image of the fabricated binary diffractive lens $(N=4)$ on the PET film. The diffractive lens, whose width was almost the same as the designed lens, was obtained.

The far-field transmitted intensity distribution of the fabricated lens is characterized by red laser light $(\lambda=635$ $\mathrm{nm})$. An aperture with a diameter of $100 \mathrm{~mm}$ was used to eliminate the light escaping from the edge of the lens. We characterize the relationship between the light distribution and the number of structures in the interval $g$ with the height of $570 \mathrm{~nm}$. Figure 6 shows the far-field transmitted intensity distribution of fabricated lenses with different values of $N(1,2,4)$. This distribution estimates the focal length of all the lenses to be approximately $160 \mu \mathrm{m}$, which is almost the same as that esti- 


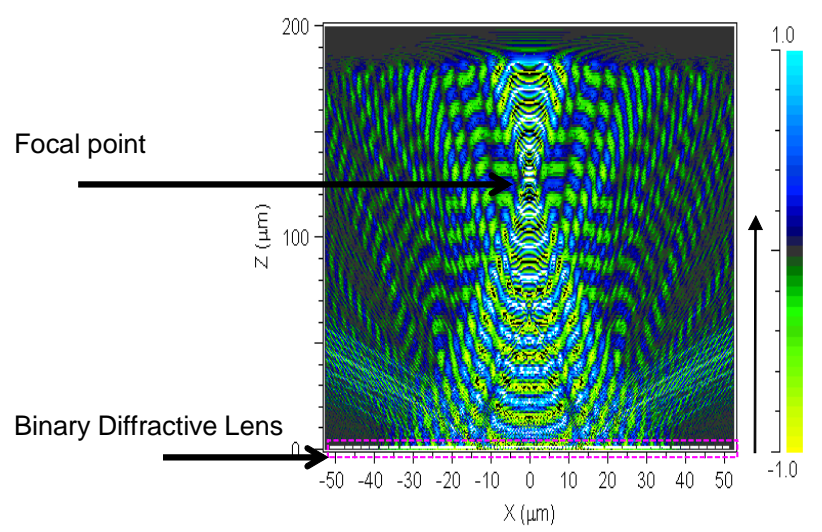

Figure 4. FDTD results for the binary diffractive convex lens.

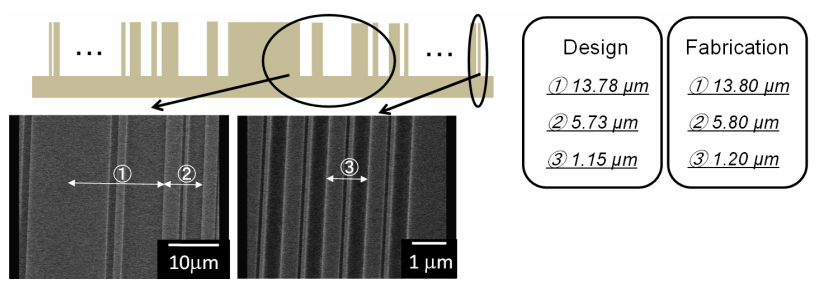

Figure 5. SEM image of the fabricated binary convex diffractive lens with a $150-\mu \mathrm{m}$ focal length $(N=4)$ on the PET film.

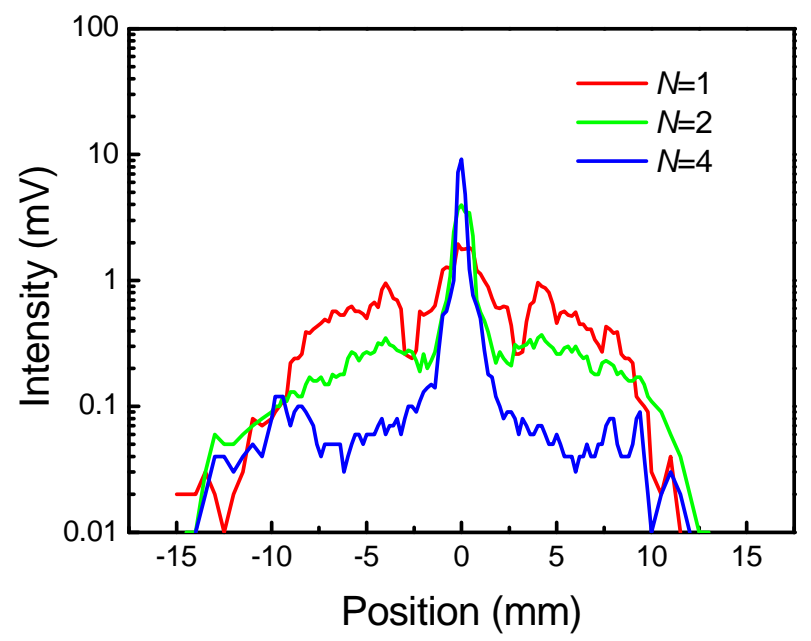

Figure 6. Far-field transmitted intensity distribution of the fabricated lens.

mated by the FDTD simulation. However, for higher $N$ values, the intensity of first-order diffraction decreases.

To determine the reason for these results, binary diffractive lenses with only first $\left(d_{1}=13.78 \mu \mathrm{m}\right)$ and 12th $\left(d_{12}=2.12 \mu \mathrm{m}\right)$ periods were fabricated. Figure 7 shows the far-field light distribution of both the lenses. In the case when $d_{1}=13.78 \mu \mathrm{m}$, first-order diffraction is observed when $N=1,2,4$. Because $d_{1}$ is considerably larger than the wavelength of light, first-order diffraction can be observed. On the other hand, in the case when $d_{12}$
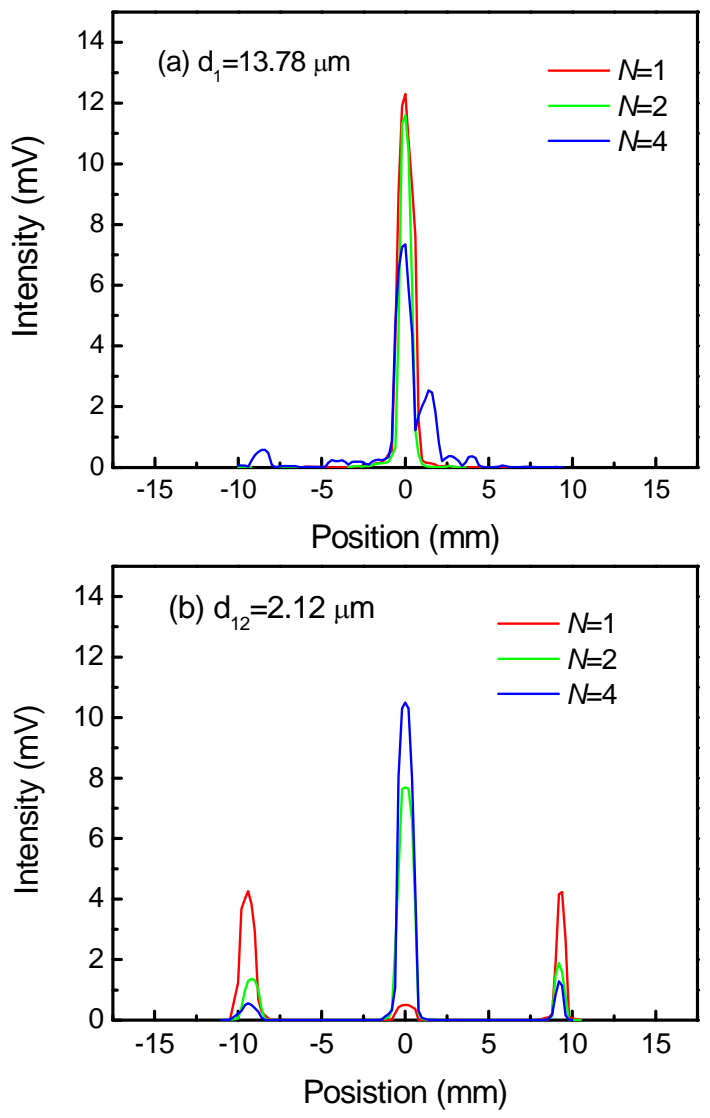

Figure 7. Far-field light distribution of the binary diffractive lenses in the case of (a) $d_{1}=13.78 \mu \mathrm{m}$ and (b) $d_{12}=2.12$ $\mu \mathrm{m}$.

$=2.12 \mu \mathrm{m}$, first-order diffraction is observed when $N=1$ and 2, and zero-order diffraction is observed when $N=4$. Therefore, to improve the diffraction efficiency of the diffractive lens, it is necessary to control the intensities of the zero- and first-order diffractions by choosing the binary structures.

On the basis of these results, to improve the first-order diffraction intensity, we fabricated a modulated structure by varying the number of approximated levels in a period. The centre part is the structure in the case when $N=1$. Moving away from the centre, $\mathrm{N}$ increases to 4 . The height of this lens is $570 \mathrm{~nm}$. The first-order diffraction intensity can be enhanced compared with diffractive lenses that have the same number of approximated levels in each period. This is because the first-order diffraction intensity is easily enhanced by each structure. Therefore, the modulated structure is effective in enhancing the first-order diffraction intensity.

\subsection{Binary Diffractive Concave Lenses}

Prior to fabrication of the binary diffractive concave lenses, the FDTD simulation is carried out to design the lens. It is confirmed that the structures of Figure 1(d) can dif- 
fuse the LED light. Figure 8 shows a scanning-electronmicroscope (SEM) image of a binary diffractive concave lens fabricated on COP film and with a $2 \mathrm{~mm}$ focal length. Circular structures were obtained. The dimensions of the fabricated lens were very close to the design specifications.

To estimate the focal length of this lens, the fabricated lens was illuminated with the green laser and the far-field light intensity distribution was recorded (see Figure 9). From the broad distribution of the laser light, the focal length of this lens was estimated to be $2 \mathrm{~mm}$. To optimize the structure of this binary diffractive concave lens, we consider how the light intensity distribution depends on the number of structures $N$ for period $d$. For large $N$, the intensity of first-order diffraction decreases because
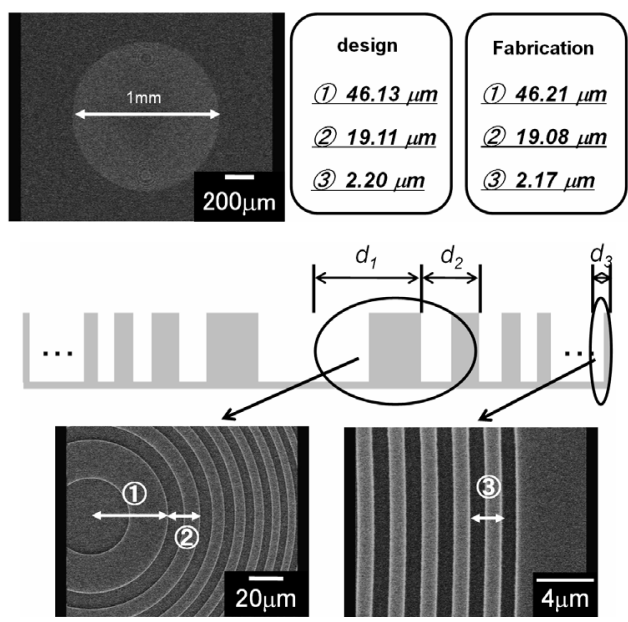

Figure 8. SEM images and specifications of fabricated binary diffractive concave lens on the COP film and with 2 mm focal length.

(a)
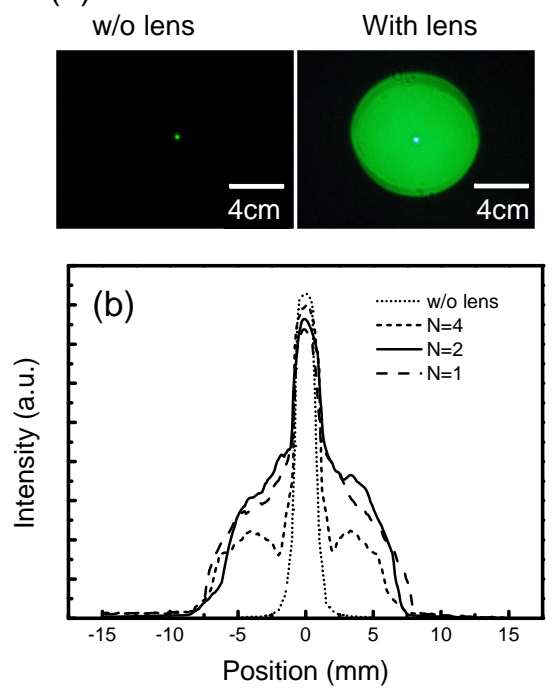

Figures 9. Photograph of (a) far-field pattern; (b) Light intensity distribution in far-field pattern. the structures, which are smaller than the wavelength, exist. Therefore, the structure must be optimized for enhancing first-order diffraction.

To apply this binary diffractive concave lens to LED lighting, we characterized it using a white-light LED. The white-light LEDs used in these experiments were surface-mount devices with light-distribution angles of $120^{\circ}$. These LEDs emit from 380 to $780 \mathrm{~nm}$ with two peaks in the spectrum: a sharp peak at $446 \mathrm{~nm}$ that is based on the peak wavelength of the blue LED and a broad peak at $555 \mathrm{~nm}$ that is based on the peak wavelength of yellow phosphor. Because of the wide spectrum, the wavelength $\lambda_{0}$ that appears in Equation (1) is not interpreted as the wavelength of a coherent light source, but is regarded as the design wavelength for the binary diffractive concave lens. For this study, we chose the design wavelengths of the binary diffractive concave lenses to be $446,500,555$, and $780 \mathrm{~nm}$, and fabricated these lenses by EBL. In this experiment, the focal length of these lenses is $0.3 \mathrm{~mm}$ because they are placed on the surface of surface mount type LEDs, and their diameter is $1.0 \mathrm{~mm}$.

To determine a suitable design wavelength $\lambda_{0}$, we characterized the chromaticity of the far-field pattern and the far-field intensity distribution for each lens. Figures 10(a) and (b) show the standard deviation of the chromaticity in the $x$ and $y$ directions for the far-field pattern. The standard deviation of the chromaticity was evaluated using the difference in chromaticity between each given far-field pattern and white

color $[(x, y)=(0.310,0.316)]$ on the chromaticity diagram, as shown in Figures 10(a) and (b). From the results of the chromaticity analysis, the colors of the farfield pattern were changed from blue to white when the design wavelength varied from 446 to $555 \mathrm{~nm}$, as shown in Figures 10(d)-(f). For a design wavelength of $555 \mathrm{~nm}$, the color of Figure 10(f) is almost same as that of Figure 10(c). When the design wavelength is $780 \mathrm{~nm}$, white light becomes red because most of the lens structures are larger than $780 \mathrm{~nm}$. For a design wavelength $\lambda_{0}=555 \mathrm{~nm}$, the standard deviation of the chromaticity is close to that of white $[(x, y)=(0.310,0.316)]$.

Next, the far-field light-distribution pattern was characterized for each lens. Figurer 11 shows the light distribution of the far-field pattern. For lenses with design wavelengths of 446 or $500 \mathrm{~nm}$, the intensity at the center of the far-field pattern is stronger than that in the surrounding areas. The chromaticity of the far-field pattern for these lenses appears blue. Therefore, we consider that blue light, which is the strongest intensity in the LED, travels straight and does not diffract. However, for lenses with design wavelengths of 555 or $780 \mathrm{~nm}$, the LED light can spread to the surrounding area. We consider that most of the LED light can be diffracted because the size 


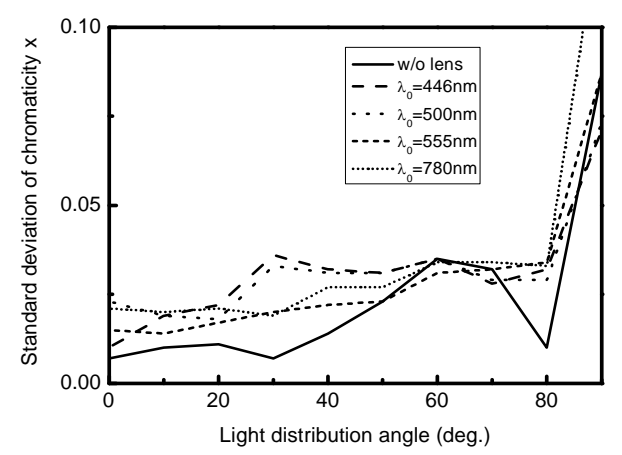

(a)

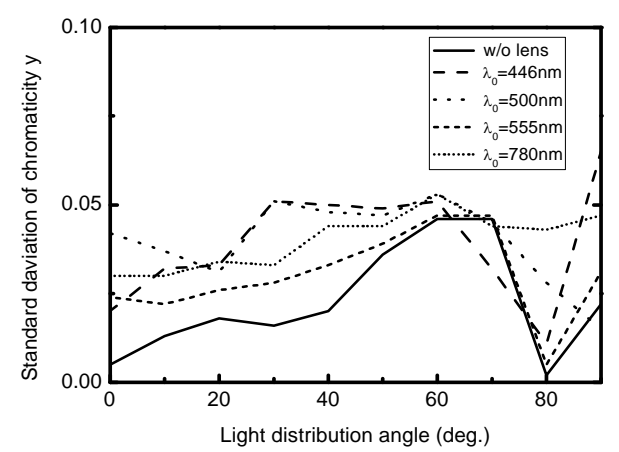

(b)

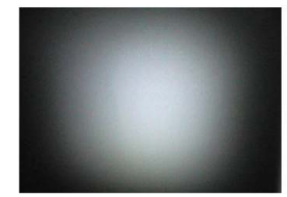

(c) w/o lens

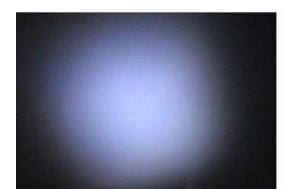

(e) $\lambda_{0}=500 \mathrm{~nm}$

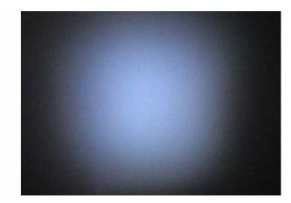

(d) $\lambda_{0}=446 \mathrm{~nm}$

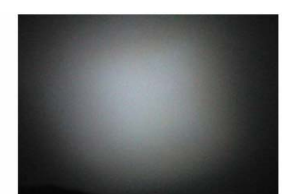

(f) $\lambda_{0}=555 \mathrm{~nm}$
Figures 10. Standard deviation of chromaticity in $x$ direction (a) and in $y$ direction (b). Photographs of far-field pattern for each design wavelength (c) without lens; (d) $\lambda_{0}=$ $446 \mathrm{~nm}$; (e) $\lambda_{0}=500 \mathrm{~nm}$; and (f) $\lambda_{0}=555 \mathrm{~nm}$.

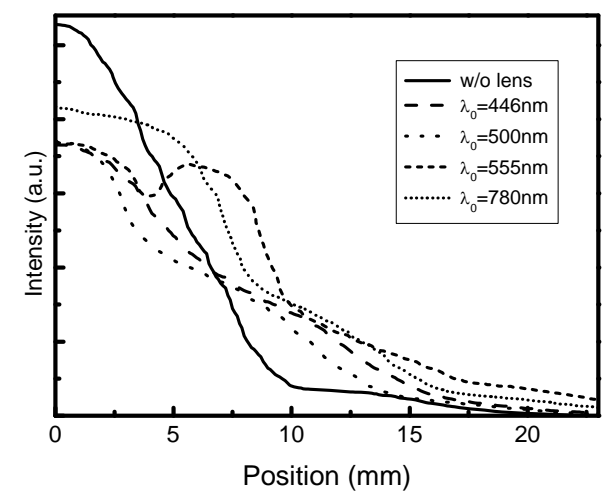

Figure 11. Far-field pattern for each design wavelength (focal length $0.3 \mathrm{~mm}$ and diameter $1.0 \mathrm{~mm}$ ). of each structure is larger than the wavelength of visible light. From these results, we determine the design wavelength $\lambda_{0}$ to be $555 \mathrm{~nm}$.

Finally, we fabricated a 2.0 -mm-diameter binary diffractive concave lens and characterized its far-field pattern. In this experiment, to enhance the diffractive light and reduce the undiffracted light, the lens structure is modulated. The center part (i.e., the first and the second period) of the lens contains fine structures $(N=4)$ and the middle part (from the third to 100th period) contains slightly rough structures $(N=2)$. The edge part (from the 101 st to the 1034th period) contains rough structures $(N$ $=1$ ). Figure 12 shows the spatial light distribution of this lens. We reduced the intensity of undiffracted light (i.e., zeroth-order diffraction) and achieved a wide first-order diffraction area $(15 \mathrm{~mm}$ diameter) for a $30 \mathrm{~mm}$ interval between the LED and photodiode.

\section{Conclusions}

In summary, we designed and fabricated two types of binary diffractive lenses using EBL on optical films film for controlling LED light. In the case of the binary diffractive convex lens with 2-mm focal length, it is possible to control the luminous intensity distribution. To improve the diffraction efficiency and realize a thin LED light source, we fabricated a binary diffractive lens with $100-\mu \mathrm{m}$-order focal length. It is clear that the intensities of the zero- and first-order diffractions are controlled by the structure of the binary diffractive lens. By using this lens, wide luminous intensity distribution can be obtained.

Furthermore we fabricated and characterized binary diffractive concave lenses for application in LED lighting. The binary diffractive concave lens fabricated in this study can be used to broadly disperse $532 \mathrm{~nm}$ light and produces the same effect as a refractive concave lens. For white-light LEDs, we chose a design wavelength of 555 $\mathrm{nm}$. By using this wavelength, aberrations are reduced and white-light LEDs are strongly diffused. Finally, we

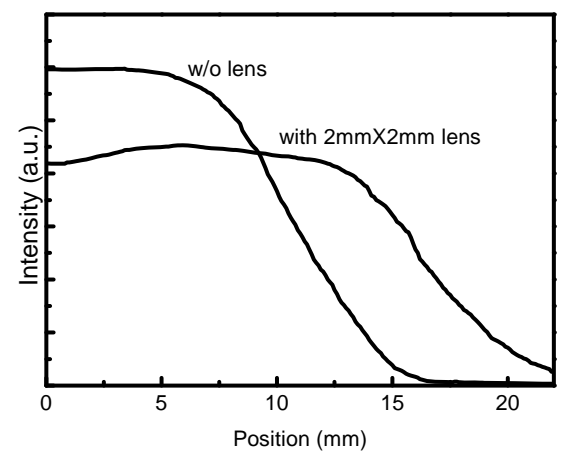

Figure 12. Far-field pattern of binary diffractive concave lens with modulated structures (focal length $0.3 \mathrm{~mm}$ and diameter $2.0 \mathrm{~mm}$ ). 
observed enhanced first-order diffraction intensity from the modulated structures.

\section{Acknowledgements}

This work was partly supported by Adaptable and Seamless Technology Transfer Program through Target-driven R\&D (A-STEP) from the Japan Science and Technology Agency (No. AS232Z00241B), a Grant-in-Aid for Scientific Research from the Japan Society for the Promotion of Science (No. 18360008, 21360007 and 2436008), Mie University COE Projects, a Start-up Grant Program for Mie Venture Business from Mie Industry Enterprise Support Center, the Kinki Invention Center, and the Knowledge Cluster Initiative from the Ministry of Education, Culture, Sports, Science and Technology.

The authors thank Mr. M. Suzuki and Mr. T. Sato (J. A. Woollam Japan Corp.) for their help with ellipsometry measurements.

The authors also thank Prof H. Miyake, Mr. K. Manabe, Mr. N. Machida, Mr. Y. Nakayama, Mr. K. Arakawa, Mr. Y. Seriguchi, K. Inoue (Mie University) and H. Yoshino (JSR Co.) for their help in the experiments and valuable discussions.

\section{REFERENCES}

[1] A. Motogaito and K. Hiramatsu, "Fabrication of Binary Diffractive Lens on Optical Films by Electron Beam Lithography," In: G. Kostovski, Ed., Unconventional Lithography, In Tech, Croatia, 2011, pp. 139-148.

[2] J. L. Soret, "Concerning Diffraction by Circular Gratings," Annual Review of Physical Chemistry, Vol. 156, 1875, pp. 99-113 (in German).

[3] L. B. Lesem, P. M. Hirsch and J. A. Jordan Jr., "The Kinoform: A New Wavefront Reconstruction Device," IBM Journal of Research and Development, Vol. 13, No. 2, 1969, pp. 150-155. doi:10.1147/rd.132.0150

[4] J. A. Jordan Jr., P. M. Hirsch, L. B. Lessem and D. L. Van Rooy, "Kinoform Lenses," Applied Optics, Vol. 9, No. 8, 1970, pp. 1883-1887.

[5] G. J. Swason and W. B. Veldkamp, "Diffractive Optical Elements for Use in Infrared Systems," Optical Engineering, Vol. 28, No. 6, 1989, pp. 605-608.

[6] Y. Orihara, W. Klaus, M. Fujino and K. Kodate, "Optimization and Application of Hybrid-Level Binary Zone
Plates," Applied Optics, Vol. 40, No. 32, 2001, pp. 5877-5885. doi:10.1364/AO.40.005877

[7] K. Yamada, W. Watanabe, Y. Li and K. Itoh, "Multilevel Phase-Type Diffractive Lenses in Silica Glass Induced by Filamentation of Femtosecond Laser Pulses," Optics Letters, Vol. 29, No. 16, 2004, pp. 1846-1848.

doi:10.1364/OL.29.001846

[8] P. Lalanne, S. Astilean, P. Chavel, E. Cambril and H. Launois, "Design and Fabrication of Blazed Binary Diffractive Elements with Sampling Periods Smaller than the Structural Cutoff," Journal of the Optical Society of America A, Vol. 16, No. 5, 1999, pp. 1143-1156. doi:10.1364/JOSAA.16.001143

[9] P. Lalanne, "Waveguiding in Blazed-Binary Diffractive Elements," Journal of the Optical Society of America A, Vol. 16, No. 10, 1999, pp. 2517-2520. doi:10.1364/JOSAA.16.002517

[10] B. H. Kleemann, M. Seesselberg and J. Rudoff, "Design Concepts for Broadband High-Efficiency DOEs," Journal of the European Optical Society, Vol. 3, 2008, Article ID: 08015. doi:10.2971/jeos.2008.08015

[11] Y. Okada, A. Murata, M. Ando, T. Suenaga, T. Korenaga and M. Suzuki, "Development a Diffraction Lens for Color Imaging Using a Nanocomposite Material with High Refractive Index and High Abbe Number," KobunshiRonbunshu, Vol. 67, No. 7, 2010, pp. 390-396 (in Japanese). doi:10.1295/koron.67.390

[12] A. Motogsito, N. Machida, T. Morikawa, K. Manabe, H. Miyake and K. Hiramatsu, "Fabrication of a Binary Diffractive Lens for Controllingthe Luminous Intensity Distribution of LED Light," Optical Review, Vol. 16, No. 4, 2009, pp. 455-457. doi:10.1007/s10043-009-0087-3

[13] A. Motogaito, K. Arakawa, Y. Nakayama, H. Miyake and K. Hiramatsu, "Fabrication and Characterization of Binary Diffractive Lens with the $100 \mu \mathrm{m}$-Order-Focal Length," Technical Digest on the 16th Microoptics Conference, Hsinchu, 31 October-3 November 2010, pp. 209-210.

[14] A. Motogaito, K. Arakawa, H. Miyake and K. Hiramatsu, "The Dependence of the 1st Order Diffraction Efficiency on the Structures of Binary Diffractive Lens with the 100 $\mu \mathrm{m}$-Order-Focal Lengths on a PET Film," Proceedings of the 2nd EOS Conference on Manufacturing of Optical Components, Munich, 23-25 May 2011.

[15] D. A. Buralli, G. M. Morris and J. R. Rogers, "Optical Performance of Holographic Kinoforms," Applied Optics, Vol. 28, No. 5, 1989, pp. 976-983. doi:10.1364/AO.28.000976 